\title{
Hepatic Differentiation of Human Induced Pluripotent Stem Cells in a Perfused Three-Dimensional Multicompartment Bioreactor
}

\author{
Nora Freyer, ${ }^{1}$ Fanny Knöspel, ${ }^{1}$ Nadja Strahl, ${ }^{1}$ Leila Amini, ${ }^{1}$ Petra Schrade, ${ }^{2}$ Sebastian Bachmann, ${ }^{2}$ \\ Georg Damm, ${ }^{3,4}$ Daniel Seehofer, ${ }^{3,4}$ Frank Jacobs, ${ }^{5}$ Mario Monshouwer, ${ }^{5}$ and Katrin Zeilinger, ${ }^{1, *}$
}

\begin{abstract}
The hepatic differentiation of human induced pluripotent stem cells (hiPSC) holds great potential for application in regenerative medicine, pharmacological drug screening, and toxicity testing. However, full maturation of hiPSC into functional hepatocytes has not yet been achieved. In this study, we investigated the potential of a dynamic threedimensional (3D) hollow fiber membrane bioreactor technology to improve the hepatic differentiation of hiPSC in comparison to static two-dimensional (2D) cultures. A total of $100 \times 10^{6}$ hiPSC were seeded into each 3D bioreactor $(n=3)$. Differentiation into definitive endoderm (DE) was induced by adding activin A, Wnt3a, and sodium butyrate to the culture medium. For further maturation, hepatocyte growth factor and oncostatin M were added. The same differentiation protocol was applied to hiPSC maintained in 2D cultures. Secretion of alpha-fetoprotein (AFP), a marker for DE, was significantly $(p<0.05)$ higher in $2 \mathrm{D}$ cultures, while secretion of albumin, a typical characteristic for mature hepatocytes, was higher after hepatic differentiation of hiPSC in 3D bioreactors. Functional analysis of multiple cytochrome P450 (CYP) isoenzymes showed activity of CYP1A2, CYP2B6, and CYP3A4 in both groups, although at a lower level compared to primary human hepatocytes (PHH). CYP2B6 activities were significantly $(p<0.05)$ higher in 3D bioreactors compared with $2 \mathrm{D}$ cultures, which is in line with results from gene expression. Immunofluorescence staining showed that the majority of cells was positive for albumin, cytokeratin 18 (CK18), and hepatocyte nuclear factor 4alpha (HNF4A) at the end of the differentiation process. In addition, cytokeratin 19 (CK19) staining revealed the formation of bile duct-like structures in 3D bioreactors similar to native liver tissue. The results indicate a better maturation of hiPSC in the 3D bioreactor system compared to 2D cultures and emphasize the potential of dynamic 3D culture systems in stem cell differentiation approaches for improved formation of differentiated tissue structures.
\end{abstract}

Keywords: stem cells; tissue engineering

\section{Introduction}

During drug development, only one out of nine compounds gets approved by the regulatory authorities, usually due to a lack of efficacy or toxic side effects. ${ }^{1}$ Thus, models for assessment of drug toxicity, especially hepatotoxicity, in the early phase of drug development are needed. Animal models, although indispensable in preclinical studies, are not sufficiently predictive for humans due to interspecies differences. ${ }^{2}$ Primary human hepatocytes $(\mathrm{PHH})$ have been widely accepted

\footnotetext{
${ }^{1}$ Bioreactor Group, Berlin Brandenburg Center for Regenerative Therapies (BCRT), Charité-Universitätsmedizin Berlin, Berlin, Germany.

${ }^{2}$ Charité Centrum Grundlagenmedizin, Institut für Vegetative Anatomie, Charité-Universitätsmedizin Berlin, Berlin, Germany.

${ }^{3}$ Department of General-, Visceral- and Transplantation Surgery, Charité-Universitätsmedizin Berlin, Berlin, Germany.

${ }^{4}$ Department of Hepatobiliary Surgery and Visceral Transplantation, University of Leipzig, Leipzig, Germany.

${ }^{5}$ Janssen Research and Development, Beerse, Belgium.

Part of this work was previously presented at the following meeting: Freyer N, Strahl N, Knöspel F, Urbaniak T, Zeilinger K. Hepatic differentiation of hiPSC in a 3D multicompartment bioreactor, 30th Annual Meeting of the German Association of the Study of the Liver (GASL), 2014, 24 and 25th January, Tübingen, Germany, abstract published in Gastroenterol 52, p. 3.12.
}

*Address correspondence to: Dr. med. vet. Katrin Zeilinger, Bioreactor Group, Berlin Brandenburg Center for Regenerative Therapies (BCRT), Charité-Universitätsmedizin Berlin, Campus Virchow-Klinikum, Augustenburger Platz 1, Berlin 13353, Germany, E-mail: katrin.zeilinger@charite.de

(c) Nora Freyer et al. 2016; Published by Mary Ann Liebert, Inc. This Open Access article is distributed under the terms of the Creative Commons License (http://creativecommons.org/licenses/by/4.0), which permits unrestricted use, distribution, and reproduction in any medium, provided the original work is properly credited. 
as the gold standard for predictive in vitro studies on hepatic drug toxicity. ${ }^{3}$ However, $\mathrm{PHH}$ display a huge variation in cell function and enzyme activities because of interdonor variances, ${ }^{4}$ and the high demand of freshly isolated $\mathrm{PHH}$ is difficult to address due to the scarce availability of human liver tissue.

Human induced pluripotent stem cells (hiPSC) represent a promising cell source for the generation of human hepatocytes for studies on hepatic drug toxicity. Due to the unlimited self-renewing capacity of hiPSC, they provide the option for cell production in large amounts and at a constant quality. In addition, variances due to genetic polymorphism can be investigated by using different hiPSC lines representative of individual patient groups. ${ }^{5}$

Several protocols have been established to generate stem cell-derived hepatocytes from human pluripotent stem cells. ${ }^{6-9}$ These procedures mimic the embryonic development of the liver by adding different growth factors necessary for each developmental stage. The resulting hepatocyte-like cells (HLC) were successfully applied for in vitro studies on human drug exposure, ${ }^{10,11}$ hepatitis $\mathrm{B}$ and $\mathrm{C}$ infection, ${ }^{12,13}$ or malaria pathogenesis ${ }^{14}$ among others, and they have been shown to repopulate the livers of chimeric mice and rescue the disease phenotype in these animals. ${ }^{15}$ However, the HLC obtained with existing protocols still show an immature phenotype with reduced hepatic functionality when compared to $\mathrm{PHH}{ }^{16,17}$

To overcome these drawbacks, improved culture models are demanded, which address the needs of the cells in their natural environment. Several studies have shown that three-dimensional (3D) culture of $\mathrm{PHH}$ in natural or synthetic scaffolds supports cell-cell contacts, cell polarization, and preservation of liver functions such as cytochrome P450 (CYP) activities, albumin production, and glycogen synthesis. ${ }^{18-20}$ To improve oxygenation and medium exchange in hepatocyte cultures, various perfused $3 \mathrm{D}$ culture systems have been developed. ${ }^{21-23}$

In the 3D multicompartment bioreactor used in this study, the cells are maintained in a perfused 3D environment allowing for physiological signal exchange and autocrine or paracrine stimulation, close to the natural situation in the organ. We have previously shown that this $3 \mathrm{D}$ bioreactor system supports stable culture of $\mathrm{PHH}$ under serum-free conditions ${ }^{24,25}$ and is suitable for differentiation of human embryonic stem cells (hESC). ${ }^{26,27}$

Thus, we hypothesize that the usage of the $3 \mathrm{D}$ bioreactor system could improve the hepatic maturation and liver-specific functionality of hiPSC-derived hepatocytes compared with conventional two-dimensional (2D) cultures. The functionality of the cells upon differentiation in $2 \mathrm{D}$ cultures or $3 \mathrm{D}$ bioreactors was evaluated by measurement of typical hepatocyte products (albumin, urea) and CYP activities. Cultures were further characterized by means of immunohistochemical investigations, transmission electron microscopy (TEM), and analysis of liver-specific mRNA expression. Data from hiPSCderived differentiated cells were compared to those from freshly isolated or $2 \mathrm{D}$ cultured $\mathrm{PHH}$.

\section{Materials and Methods}

Bioreactor technology

The 3D multicompartment bioreactor consists of three independent, but interwoven hollow fiber capillary systems that serve for counter-current medium perfusion (two medium compartments). Cells are supplied with oxygen by direct membrane oxygenation through integrated gas capillaries (gas compartment), which are perfused with an air/ $\mathrm{CO}_{2}$ mixture. Cells are cultured in the extracapillary space (cell compartment). The analytical scale bioreactors used in this study have a cell compartment volume of $2 \mathrm{~mL}$. A detailed description of the technology is provided elsewhere. ${ }^{28}$

Bioreactors are operated in a perfusion device with two modular pump units, one for medium recirculation and one for medium feed. The bioreactor incubation chamber is heated by two heating units located inside the chamber, each consisting of a heating cartridge and a fan. A platinum measuring resistor monitors the temperature inside the chamber and software is used to set and maintain the desired temperature. Gas flow rates and gas compositions are regulated using electronically operated gas valves for air, $\mathrm{CO}_{2}$, and the resulting gas mixture (Vögtlin Instruments). Bioreactors, tubing systems, and perfusion devices were manufactured by Stem Cell Systems.

\section{Hepatic differentiation of hiPSC in 3D bioreactors} or 2D cultures

The hiPSC line DF6-9-9T ${ }^{29}$ (WiCell Research Institute) was cultured under feeder-free conditions on Nunclon $^{\mathrm{TM}}$ six-well cell culture plates (ThermoScientific Nunc $^{\mathrm{TM}}$ ) coated with $8.68 \mu \mathrm{g} / \mathrm{cm}^{2}$ Matrigel (growth factor reduced). Cells were expanded with the $\mathrm{mTeSR}^{\mathrm{TM}} 1$ medium (StemCell Technologies) with $0.05 \mathrm{mg} / \mathrm{mL}$ gentamicin (Merck). Afterward, a total of $100 \times 10^{6}$ hiPSC were seeded into a precoated bioreactor $(8.68 \mu \mathrm{g} /$ $\mathrm{cm}^{2}$; Matrigel) and cultured over a total of 20 days. 
Bioreactors were maintained at $37^{\circ} \mathrm{C}$, the medium recirculation rate was $10 \mathrm{~mL} / \mathrm{min}$, and the feed rate was $1 \mathrm{~mL} / \mathrm{h}$. Based on daily measurements of the $\mathrm{pH}$, glucose, and lactate values, $\mathrm{CO}_{2}$ and medium perfusion rates were adjusted, if necessary, to maintain a stable $\mathrm{pH}$ between 7.2 and 7.4 and sufficient glucose levels (>25 mg/dL).

After a proliferation phase of 3 days with $\mathrm{mTeSR}^{\mathrm{TM}} 1$, differentiation of the cells in 3D bioreactors was induced based on the protocols described by Hay et al. ${ }^{6,30,31}$ for $2 \mathrm{D}$ cultures. In the first step, differentiation into definitive endoderm (DE) was induced by perfusion with the Roswell Park Memorial Institute (RPMI) 1640 culture medium (Merck) supplemented with $100 \mathrm{ng} / \mathrm{mL}$ activin A (Peprotech), $50 \mathrm{ng} / \mathrm{mL}$ Wnt3a (R\&D Systems), $1 \mu \mathrm{M}$ sodium butyrate (Sigma-Aldrich), and 2\% (v/v) B27 supplements without insulin (Life Technologies) for 3 days.

Subsequently, bioreactors were perfused over 13 days with a hepatocyte culture medium consisting of basal medium and single quots (Lonza) and $10 \mathrm{ng} / \mathrm{mL}$ hepatocyte growth factor (HGF; Peprotech) to induce differentiation of DE-cells to hepatoblasts. For further maturation to HLC, $10 \mathrm{ng} / \mathrm{mL}$ oncostatin M (Peprotech) was added during the last 4 days of differentiation.

$2 \mathrm{D}$ cultures were performed in parallel with $3 \mathrm{D}$ bioreactor cultures for control, applying the same differentiation protocol with daily medium exchange.

\section{Metabolic parameters}

The metabolic activity of the cells was assessed by daily measurement of glucose and lactate concentrations with a blood gas analyzer (ABL 700; Radiometer). Potential cell damage was detected by analyzing the release of lactate dehydrogenase (LDH) using an automated clinical chemistry analyzer (Cobas ${ }^{\circledR}$ 8000; Roche Diagnostics) as well as the production of urea and the albumin precursor protein alpha-fetoprotein (AFP). Albumin secretion, as a marker for mature hepatocytes, was detected using an ELISA Quantitation kit and tetramethylbenzidine (TMB) substrate (both from Bethyl Laboratories) according to the manufacturer's instructions.

\section{Culture of $\mathrm{PHH}$}

The PHH were isolated from macroscopically healthy tissue that remained from resected human liver of patients with primary or secondary liver tumors or benign local liver diseases. Informed consent of the patients for the use of tissue for research purposes was obtained according to the ethical guidelines of the Charité-Universitätsmedizin Berlin. Part of the tissue sample was fixed in formaldehyde for immunofluorescence staining. Cell isolation was performed according to Pfeiffer et al. ${ }^{32}$

Hepatocytes were seeded at a density of $2.0 \times 10^{5}$ cells $/ \mathrm{cm}^{2}$ in six-well plates (BD Sciences) coated with rat tail collagen. Cells were cultivated using Heparmed Vito 143 supplemented with $10 \%$ fetal calf serum (PAA), $0.8 \mathrm{mg} / \mathrm{mL}$ insulin, $5 \mathrm{mg} / \mathrm{L}$ transferrin, $0.003 \mathrm{mg} /$ L glucagon, $100 \mathrm{U} / \mathrm{mL}$ penicillin, and $100 \mu \mathrm{g} / \mathrm{mL}$ streptomycin (all Merck).

\section{Measurement of cytochrome P450 (CYP) isoenzyme activities}

Activities of the pharmacologically relevant CYP isoenzymes CYP1A2, CYP2B6, CYP2C9, and CYP3A4 were measured in (1) undifferentiated hiPSC, (2) HLC differentiated in 2D cultures or (3) 3D bioreactors, and (4) $\mathrm{PHH}$ cultures ( $24 \mathrm{~h}$ after seeding) serving as control. The cells were incubated with a cocktail containing phenacetin (Sigma) as a substrate for CYP1A2, bupropion (Toronto Research Chemicals) as a substrate for CYP2B6, diclofenac as a substrate for CYP2C9, and midazolam as a substrate for CYP3A4/5 (both from Sigma-Aldrich). Samples were taken at 1,2, 4, and $6 \mathrm{~h}$ subsequent to substrate application. An overview of the used substrates, their final concentrations, and the corresponding CYP isoenzymes is provided in Table 1.

Formed metabolites (acetaminophen, 6-OH-bupropion, 4-OH-diclofenac, 1-OH-midazolam) were quantified by liquid chromatography tandem-mass spectrometry. Deuterated 1-OH-midazolam was added as an internal standard. Separation was carried out using an Acquity UPLC C18 column and eluted fractions were directly passed through a Xevo TQ-S tandem mass

Table 1. CYP Isoenzymes Tested and Their Corresponding Substrates with Resulting Products and Applied Concentrations

\begin{tabular}{lllcr}
\hline Enzyme & Substrate & \multicolumn{1}{c}{ Product } & Final concentration $[\mu \mathrm{M}]$ & Transition reactions for analysis of probe products \\
\hline CYP1A2 & Phenacetin & Acetaminophen & 100 & $152 \rightarrow 110$ \\
CYP2B6 & Bupropion & 6-Hydroxybupropion & 500 & $256 \rightarrow 238$ \\
CYP2C9 & Diclofenac & 4'-Hydroxydiclofenac & 25 & $312 \rightarrow 266$ \\
CYP3A4/5 & Midazolam & 1'-Hydroxymidazolam & 10 & $342 \rightarrow 324$
\end{tabular}


Table 2. Applied Biosystems TaqMan Gene Expression Assays $^{\circledR}$

\begin{tabular}{|c|c|c|}
\hline $\begin{array}{l}\text { Gene } \\
\text { symbol }\end{array}$ & Gene name & Assay ID \\
\hline POU5F1 & POU domain, class 5 , transcription factor 1 & HS00999632_g1 \\
\hline NANOG & Nano & HS02387400_g1 \\
\hline SOX7 & SRY-box 7 & HS00846731_s1 \\
\hline SOX17 & SRY-box 17 & HS00751752_s1 \\
\hline AFP & Alpha fetoprotein & HS00173490_m1 \\
\hline$A L B$ & Albumin & HS00910225_m1 \\
\hline CYP1A2 & $\begin{array}{c}\text { Cytochrome P450 family } 1 \\
\text { subfamily A member } 2\end{array}$ & HS00167927_m1 \\
\hline CYP2B6 & $\begin{array}{c}\text { Cytochrome P450 family } 2 \\
\text { subfamily B member } 6\end{array}$ & HS03044634_m1 \\
\hline CYP2C9 & $\begin{array}{c}\text { Cytochrome P450 family } 3 \\
\text { subfamily A member } 4\end{array}$ & HS00426397_m1 \\
\hline CYP3A4 & $\begin{array}{c}\text { Cytochrome P450 family } 3 \\
\text { subfamily A member } 4\end{array}$ & HS00604506_m1 \\
\hline GATA2 & GATA binding protein 2 & 19_m1 \\
\hline NEFL & Neurofilament, light polypeptide & HS00196245_m1 \\
\hline GAPDH & Glyceraldehyde-3-phosphate dehydrogenase & HS03929097_g1 \\
\hline
\end{tabular}

spectrometer (both from Waters Corp.). Acquired data were processed with Thermo Xcaliber 20 software (Thermo Scientific).

\section{Gene expression analysis}

RNA was isolated from undifferentiated hiPSC, from HLC differentiated in 2D cultures or 3D bioreactors, and from freshly isolated PHH serving as reference cells. RNA isolation and subsequent cDNA synthesis were performed as described elsewhere. ${ }^{33}$ Each cDNA template was mixed with polymerase chain reaction (PCR) Master mix (Applied Biosystems) and human-specific primers and probes (TaqMan Gene Expression Assay system; Life Technologies; Table 2). Quantitative real-time PCR (qRT-PCR) was performed using a Real time cycler (Mastercycler ep Realplex 2; Eppendorf). The expression of specific genes was normalized to that of the house- keeping gene glyceraldehyde-3-phosphate dehydrogenase (GAPDH) and fold changes of expression levels were calculated with the $\Delta \Delta \mathrm{Ct}$ method. ${ }^{34}$

\section{Immunofluorescence studies}

Immunofluorescence staining was performed with antibodies listed in Table 3 as described elsewhere ${ }^{33}$ with following changes for $3 \mathrm{D}$ and tissue sections. The hollow fiber bed was excised en-bloc, fixed with $4 \%$ formaldehyde solution (Herbeta Arzneimittel), dehydrated, paraffinized, and cut into slides of $2.5 \mu \mathrm{m}$ thickness. Subsequently, the slides were deparaffinized, rehydrated, and subjected to antigen retrieval in a citrate buffer $(\mathrm{pH}$ 6.0) in a pressure cooker for $15 \mathrm{~min}$. The same procedure was applied to native human liver tissue serving as a positive control. Nuclei in native human liver tissue were stained with bisBenzimide H 33342 trihydrochloride (Sigma).

$\mathrm{Ki}-67$ positive cells were quantified in undifferentiated hiPSC, 2D cultured DE cells, and HLC from 2D cultures or $3 \mathrm{D}$ bioreactors with the open source image processing program ImageJ using at least 10 randomly chosen visual fields for each group.

\section{Transmission electron microscopy}

For TEM, part of the 3D bioreactor cell compartment was fixed with $2.5 \%$ glutaraldehyde in $0.1 \mathrm{M}$ sodium cacodylate buffer (both from Serva) over night, followed by postfixing in $1 \% \mathrm{OsO}_{4}$ (Science Services) with $0.8 \%$ potassium ferrocyanide (Merck) in $0.1 \mathrm{M}$ sodium cacodylate buffer for $1.5 \mathrm{~h}$. Subsequently, samples were progressively dehydrated in ethanol and then embedded in Epon (Serva). Ultrathin sections were stained with uranyl acetate and Reynold's lead citrate

Table 3. Antibodies Used for Immunofluorescence Staining

\begin{tabular}{|c|c|c|c|c|c|}
\hline & Protein symbol & Species & Manufacturer & Cat.-No. & Final conc. $[\mu \mathrm{g} / \mathrm{mL}]$ \\
\hline \multicolumn{6}{|l|}{ Primary antibody } \\
\hline Alpha fetoprotein & AFP & mouse & Santa Cruz & Sc-8399 & 2 \\
\hline Albumin & ALB & mouse & Sigma & A6684 & 67 \\
\hline Cytokeratin 18 & CK18 & mouse & Santa Cruz & Sc-6259 & 2 \\
\hline Cytokeratin 19 & CK19 & rabbit & Santa Cruz & Sc-25724 & 2 \\
\hline Cytochrome P450 1A2 & CYP1A2 & mouse & Santa Cruz & Sc-53614 & 2 \\
\hline Cytochrome P450 2B6 & CYP2B6 & rabbit & Santa Cruz & Sc-67224 & 2 \\
\hline Hepatocyte nuclear factor 4 alpha & HNF4A & rabbit & Santa Cruz & Sc-8987 & 2 \\
\hline Marker of proliferation $\mathrm{Ki}-67$ & MKI67 & mouse & BD Biosciences & 556003 & 10 \\
\hline Multidrug resistance-associated protein 2 & MRP2 & rabbit & Sigma & M8316 & 9 \\
\hline POU domain, class 5, transcription factor 1 & OCT3 & rabbit & Santa Cruz & Sc-9081 & 2 \\
\hline Stage-specific embryonic antigen 4 & SSEA4 & mouse & R\&D Systems & MAB1435 & 10 \\
\hline Tight junction protein 1 & TJP1 & mouse & LSBio & LS B2228 & 30 \\
\hline \multicolumn{6}{|l|}{ Secondary antibody } \\
\hline Alexa Fluor ${ }^{\circledR} 488$ anti-mouse & & goat & Life Technologies & A-11029 & 2 \\
\hline Alexa Fluor 594 anti-rabbit & & goat & Life Technologies & A-11037 & 2 \\
\hline
\end{tabular}

Final concentrations are given in $\mu \mathrm{g} / \mathrm{mL}$. 
(Merck) and microphotographs were taken using an electron microscope EM 906 (Carl Zeiss).

\section{Statistical evaluation}

Experiments were performed in triplicates, unless stated otherwise, and results are presented as mean \pm standard error of the mean.

The area under curve (AUC) was calculated for time courses of biochemical parameters and differences between culture systems were detected with a subsequent two-tailed Student's $t$-test. CYP activities of undifferentiated hiPSC were compared with those of HLC from $2 \mathrm{D}$ cultures or $3 \mathrm{D}$ bioreactors by one-way analysis of variance (ANOVA) followed by Bonferroni's multiple comparison test. Differences in gene expression between both culture systems were detected by applying an unpaired, two-tailed Student's $t$-test. $\mathrm{PHH}$ were compared with all other groups by one-way ANOVA followed by Dunnett's multiple comparison test. Differences were judged as significant if the $p$-value was $<0.05$.

\section{Results}

Metabolic activity and integrity of HLC in 2D

cultures or 3D bioreactors

In both culture systems, the metabolic activity of the cells during hepatic differentiation, assessed by glucose consumption and lactate production, showed an increase over time (Fig. 1A, B). Average glucose consumption rates were significantly higher $(p \leq 0.05)$ in $2 \mathrm{D}$ cultures than in $3 \mathrm{D}$ bioreactors (Fig. 1A). The time course of lactate production reflected that of glucose consumption (Fig. 1B). LDH release, indicating cell injury, was significantly higher $(p \leq 0.01)$ in $2 \mathrm{D}$ cultures than in 3D bioreactors during the first 3 days of differentiation, but decreased from day 3 on to basal levels. In contrast, $\mathrm{LDH}$ release in $3 \mathrm{D}$ bioreactors remained on a basal level over the whole culture period (Fig. 1C).

\section{Secretion of stage-specific proteins and metabolites}

The synthesis of liver-specific proteins and the activity of the urea cycle were evaluated during hepatic differentiation. The endoderm-specific marker AFP showed an increase during the hepatoblast differentiation stage, starting on day 7 in $2 \mathrm{D}$ cultures and on day 9 in $3 \mathrm{D}$ bioreactors, but the increase was significantly higher $(p \leq 0.05)$ in 2D cultures (Fig. 1D). Albumin also showed an increase during the hepatoblast differentiation phase beginning on day 12 of differentiation in $2 \mathrm{D}$ cultures and 3D bioreactors. However, maximum values achieved were thrice higher in $3 \mathrm{D}$ bioreactors than in $2 \mathrm{D}$ cultures (Fig. 1E), although the difference was not significant.
Values of urea secretion showed a constant increase in $3 \mathrm{D}$ bioreactors until day 17 to the threefold of secretion rates detected on day 1 of culture. A fluctuating time course was observed in $2 \mathrm{D}$ cultures with peaks at the beginning of culture and between day 9 and 13 (Fig. 1F). Values approximated those of 3D bioreactors during the final days of differentiation.

\section{Functional analysis of different cytochrome} P450 (CYP) isoenzymes

To investigate the CYP activity, the ability of the HLC to metabolize various substrates into their isoenzymespecific products was analyzed and compared with CYP activities of undifferentiated hiPSC and those of $\mathrm{PHH}$ cultures determined $24 \mathrm{~h}$ after seeding.

CYP1A2 showed a higher activity in 3D bioreactors than in 2D cultures, while it was lower compared with undifferentiated hiPSC or PHH (Fig. 2A). A significant CYP2B6-dependent conversion of bupropion to 6-OH-bupropion was observed in $3 \mathrm{D}$ bioreactors compared to $2 \mathrm{D}$ cultures ( $p \leq 0.05$ Fig. $2 \mathrm{~B}$ ). In contrast, CYP3A4 showed higher activities in undifferentiated hiPSC and in 2D cultures than in 3D bioreactors, although the differences were not significant (Fig. 2C). The isoenzyme CYP2C9 activity was detected neither in differentiated nor in undifferentiated hiPSC (data not shown). All enzyme activities investigated were significantly higher in $\mathrm{PHH}$ than in the HLC generated from hiPSC in $2 \mathrm{D}$ or $3 \mathrm{D}$ cultures (Fig. $2 \mathrm{~A}-\mathrm{C}$ ).

Gene expression of pluripotency factors, and endodermal and hepatic markers

The expression of 12 stage-specific genes was comparatively analyzed by qRT-PCR in $2 \mathrm{D}$ or $3 \mathrm{D}$ cultures of HLC and in freshly isolated PHH (Fig. 3). Expression levels were plotted relative to those of undifferentiated hiPSC (d0) to investigate the hepatic maturation state of the cells.

The expression of the pluripotency markers POU5F1 and NANOG decreased in both culture systems compared to undifferentiated hiPSC, but the decrease was significantly higher in $2 \mathrm{D}$ cultures $(p \leq 0.05$, Fig. $3 \mathrm{~A}$, B). PHH showed POU5F1 levels similar to HLC in 2D cultures, while NANOG expression was similar to those in 3D bioreactors (Fig. 3A, B).

To exclude differentiation into nonendodermal cells, markers for mesoderm (GATA2) and endoderm (neurofilament, NEFL) were analyzed as well. The analysis revealed a $>100$-fold increase for GATA2 in 3D bioreactors, which is significantly higher than in $2 \mathrm{D}$ cultures showing an $\sim 20$-fold increase (Fig. 3C). The expression 
A Glucose consumption

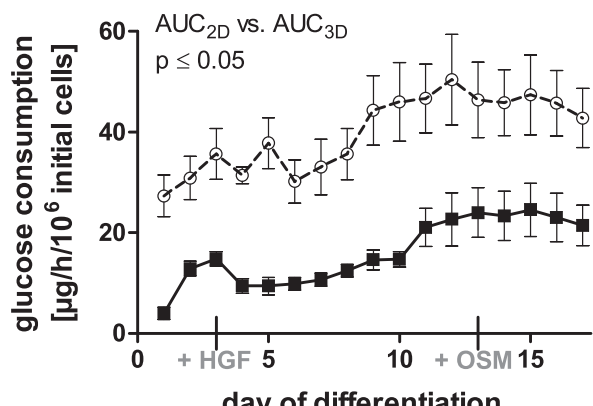

C

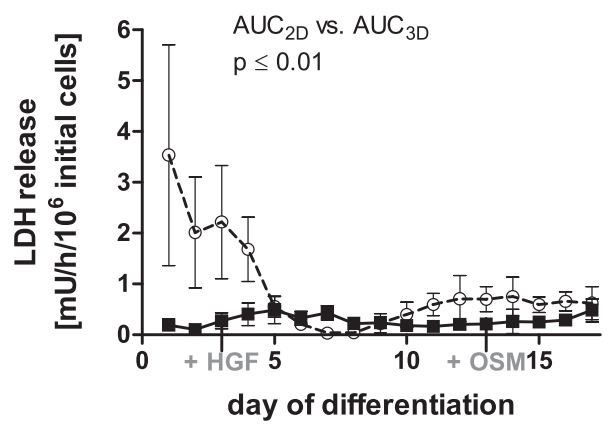

E

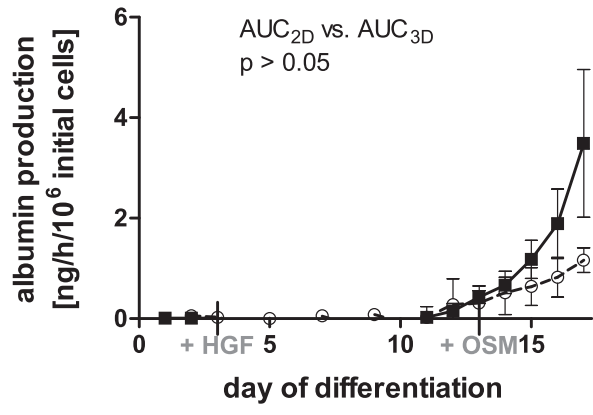

$-\Im \cdot 2 D$ cultures

\section{B Lactate production}

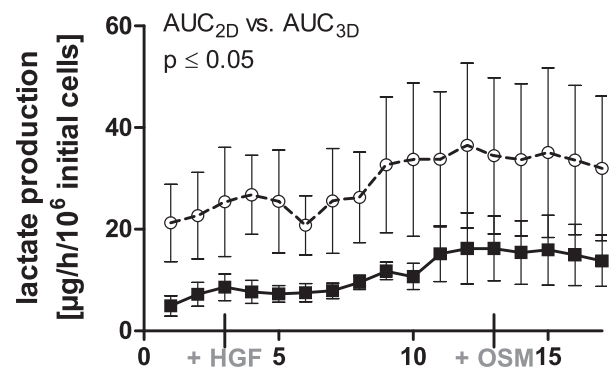

day of differentiation
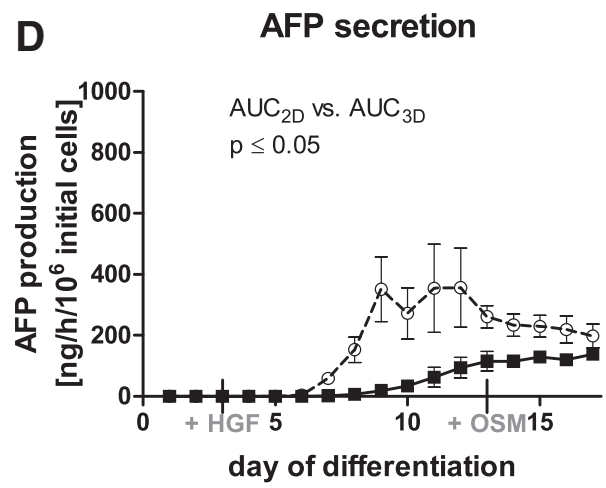

F

Urea secretion

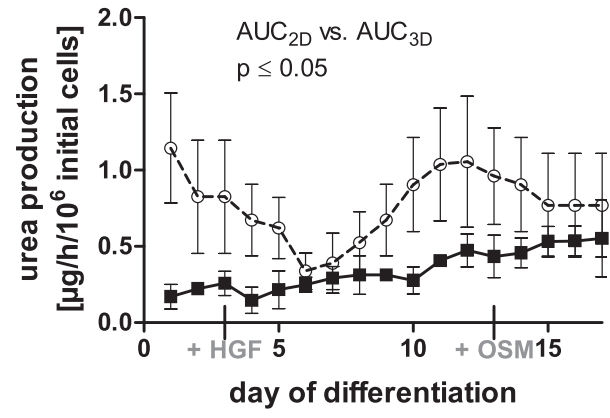

$3 \mathrm{D}$ bioreactors

FIG. 1. Metabolic activity of hiPSC during hepatic differentiation in 2D cultures (dotted line) or in 3D bioreactor cultures (black line). (A) Glucose consumption, (B) lactate production, (C) release of LDH, (D) secretion of AFP, (E) albumin production, and (F) urea secretion. Values were normalized to $1 \times 10^{6}$ inoculated cells. AUC was calculated and differences were detected with the unpaired, two-tailed Student's $t$-test (3D bioreactors: $n=3,2 \mathrm{D}$ cultures: $n=4$, mean \pm SEM). AFP, alpha-fetoprotein; AUC, area under curve; 2D, two dimensional; 3D, three dimensional; hiPSC, human induced pluripotent stem cells; LDH, lactate dehydrogenase; SEM, standard error of the mean. 


\section{A}
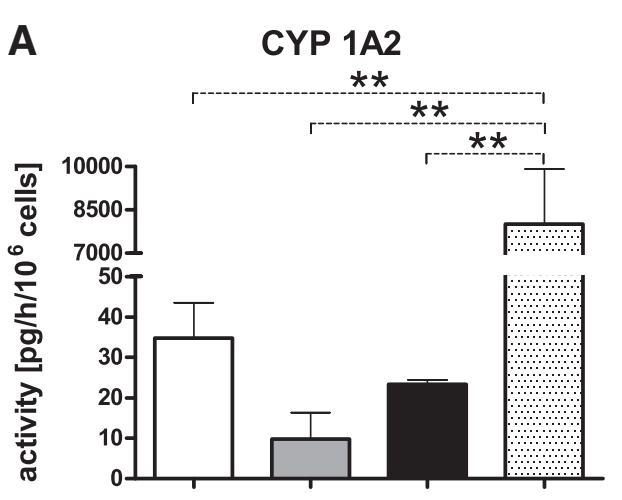

C CYP 3A4/5

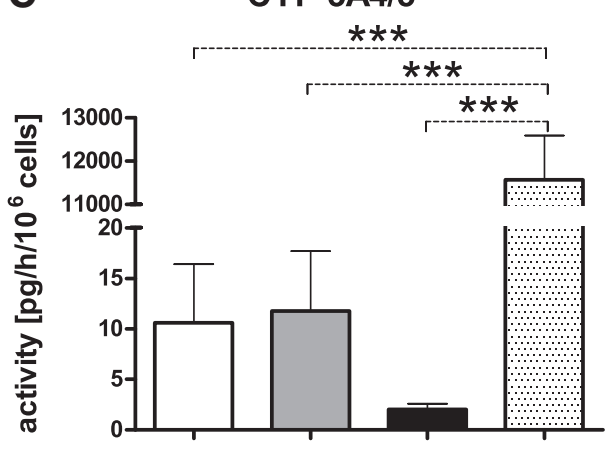

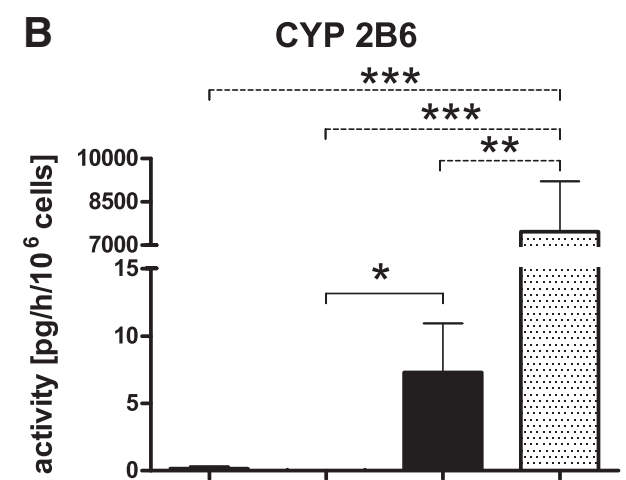

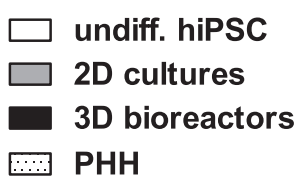

FIG. 2. Activities of different cytochrome $P 450$ (CYP) isoenzymes in undifferentiated hiPSC (white), in hiPSC after hepatic differentiation in 2D cultures (gray) or 3D bioreactors (black), or in PHH (dotted). CYP activities were determined by measuring the conversion rates of selected substrates into isoenzyme-specific products. (A) Formation of acetaminophen from phenacetin by CYP1A2, (B) formation of 6-OH-bupropion from bupropion by CYP2B6, and (C) formation of 1-OH-midazolam from midazolam by CYP3A4/5. Differences in metabolic activity between undifferentiated hiPSC, 2D cultures and 3D bioreactors, were calculated using oneway ANOVA with Bonferroni's multiple comparison test (solid line). In addition, differences between PHH to all other groups were calculated using one-way ANOVA with Dunnett's multiple comparison test (dotted line). (3D bioreactors: $n=3,2 \mathrm{D}$ cultures and undifferentiated hiPSC: $n=4$, PHH: $n=5$; mean \pm SEM), ${ }^{*} p \leq 0.05,{ }^{* *} p \leq 0.01$, ${ }^{* * *} p \leq 0.001$. ANOVA, analysis of variance; PHH, primary human hepatocytes.

of NEFL did not change significantly in comparison to undifferentiated hiPSC (Fig. 3D). PHH showed only scarce expression of NEFL and GATA2 (Fig. 3C, D).

In contrast, most markers for endoderm and mature hepatocytes increased in both culture systems. AFP showed a $>60,000$-fold increased expression in both $2 \mathrm{D}$ cultures and 3D bioreactors, relative to undifferentiated hiPSC (Fig. 3E), and the expression of SOX17 (Fig. 3F), another endodermal marker, showed a 20 -fold increase, while $\mathrm{PHH}$ showed no relevant expression of those markers. Since AFP and SOX17 are markers for both, definitive and extraembryonic endoderm, SOX7 (Fig. 3G) was analyzed as a specific marker for extraembryonic endoderm. The expression of SOX7 was increased in both culture systems and was similar to that detected in PHH.

Among the markers investigated for mature hepatocytes, the highest increase was detected for albumin $(A L B)$, which was increased in both groups by $>2,000$ fold, with $3 \mathrm{D}$ bioreactors showing twice the expression compared to 2D cultures (Fig. 3H).

In contrast, the expression of CYP1A2 was decreased compared with undifferentiated hiPSC both in 2D cultures and 3D bioreactors (Fig. 3I), which is in line with the results from activity measurements (Fig. 2A). A decrease of mRNA expression was also detected for CYP2B6, but the expression was still significantly higher in $3 \mathrm{D}$ bioreactors ( $p \leq 0.05$, Fig. $3 \mathrm{~J})$ and is in accordance to 
A
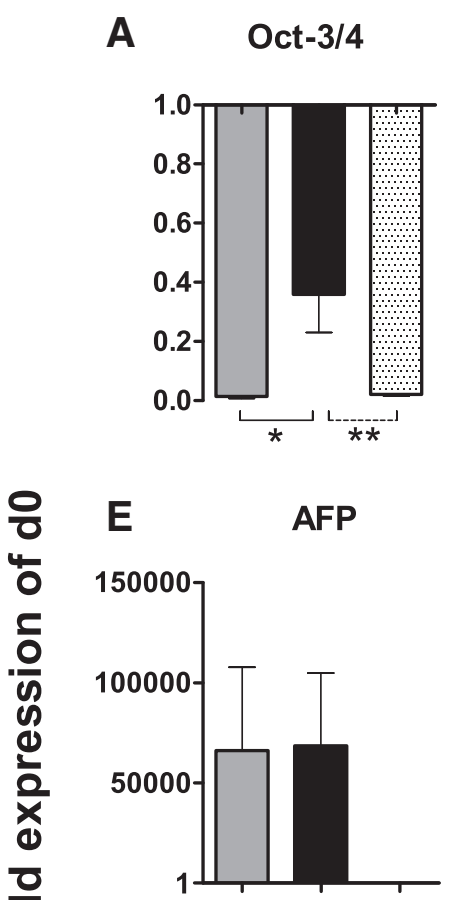

CYP1A2

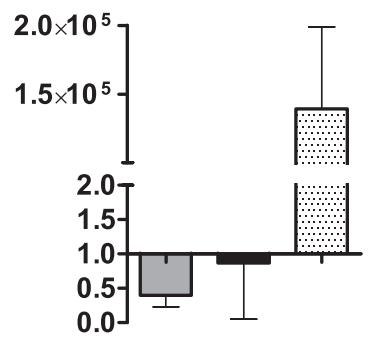

2D cultures
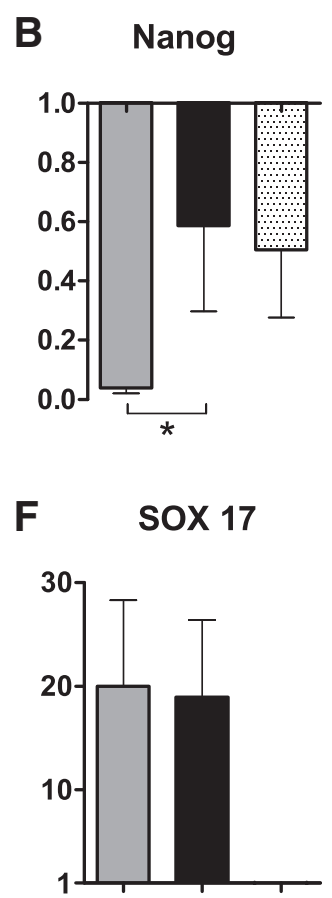

G

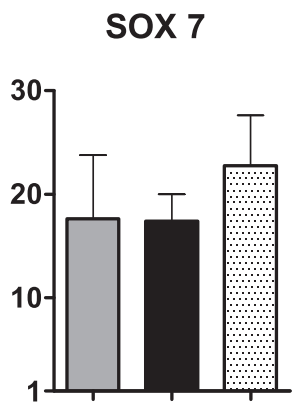

$\mathbf{J}$
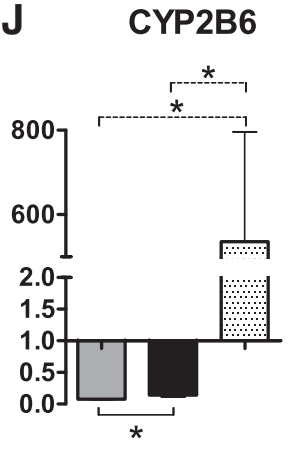

\section{D bioreactors}

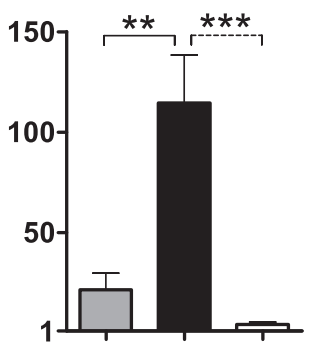

D Neurofilament
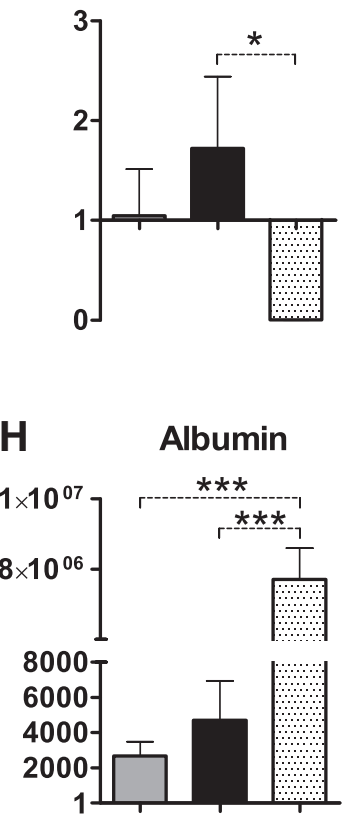

$\mathbf{L}$
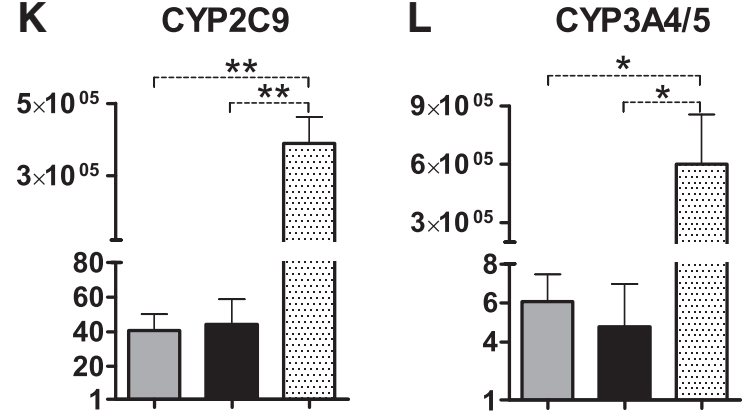

PHH

FIG. 3. Gene expression of pluripotency markers (A) Oct-3/4 and (B) Nanog, mesodermal marker (C) GATA-2, ectodermal marker (D) neurofilament, endodermal markers (E) AFP and (F) SOX 17, extra-embryonic marker (G) SOX 7 and hepatic markers (H) albumin, (I) CYP1A2, (J) CYP2B6, (K) CYP2C9 and (L) CYP3A4/5 in hiPSC after hepatic differentiation in 2D cultures or 3D bioreactors, and in $\mathrm{PHH}$ relative to undifferentiated hiPSC (d0). Samples for mRNA expression analysis were taken after hepatic differentiation of hiPSC in 2D cultures (black) or 3D bioreactors (gray). For mRNA expression analysis of PHH (dotted), freshly isolated cells were used. Fold changes relative to undifferentiated hiPSC were calculated with normalization to GAPDH expression by the $\Delta \Delta C t$ method. Differences in gene expression between 2D cultures and 3D bioreactors were calculated using the unpaired, two-tailed Student's $t$-test (solid line). In addition, differences between PHH and all other groups were calculated by means of one-way ANOVA with Dunnett's multiple comparison test (dotted line) (3D bioreactors: $n=3,2 \mathrm{D}$ cultures and undifferentiated hiPSC: $n=4, \mathrm{PHH}: n=3$; mean $\pm \mathrm{SEM}$ ), ${ }^{*} p \leq 0.05,{ }^{* *} p \leq 0.01$, ${ }^{* * *} p \leq 0.001$. 
the findings from activity measurements (Fig. 2B). Expression of CYP2C9 and CYP3A4/5 was increased by around 40- or five-fold, respectively, compared with undifferentiated cells showing no significant differences between 2D cultures and 3D bioreactors (Fig. 3K, L). All markers for mature hepatocytes showed a distinctly lower expression in HLC than in $\mathrm{PHH}$, irrespective of the culture system.
Immunohistochemical characterization of hiPSC-derived differentiated cells

To determine the amount of proliferating cells before, during, and after the hepatic differentiation of hiPSC, an immunofluorescence staining with the proliferation marker Ki-67 was performed. In cultures of undifferentiated hiPSC, almost all cells $(97.7 \% \pm 1.6 \%)$ were positive for Ki-67 (Fig. 4A1). In 2D cultured DE cells,

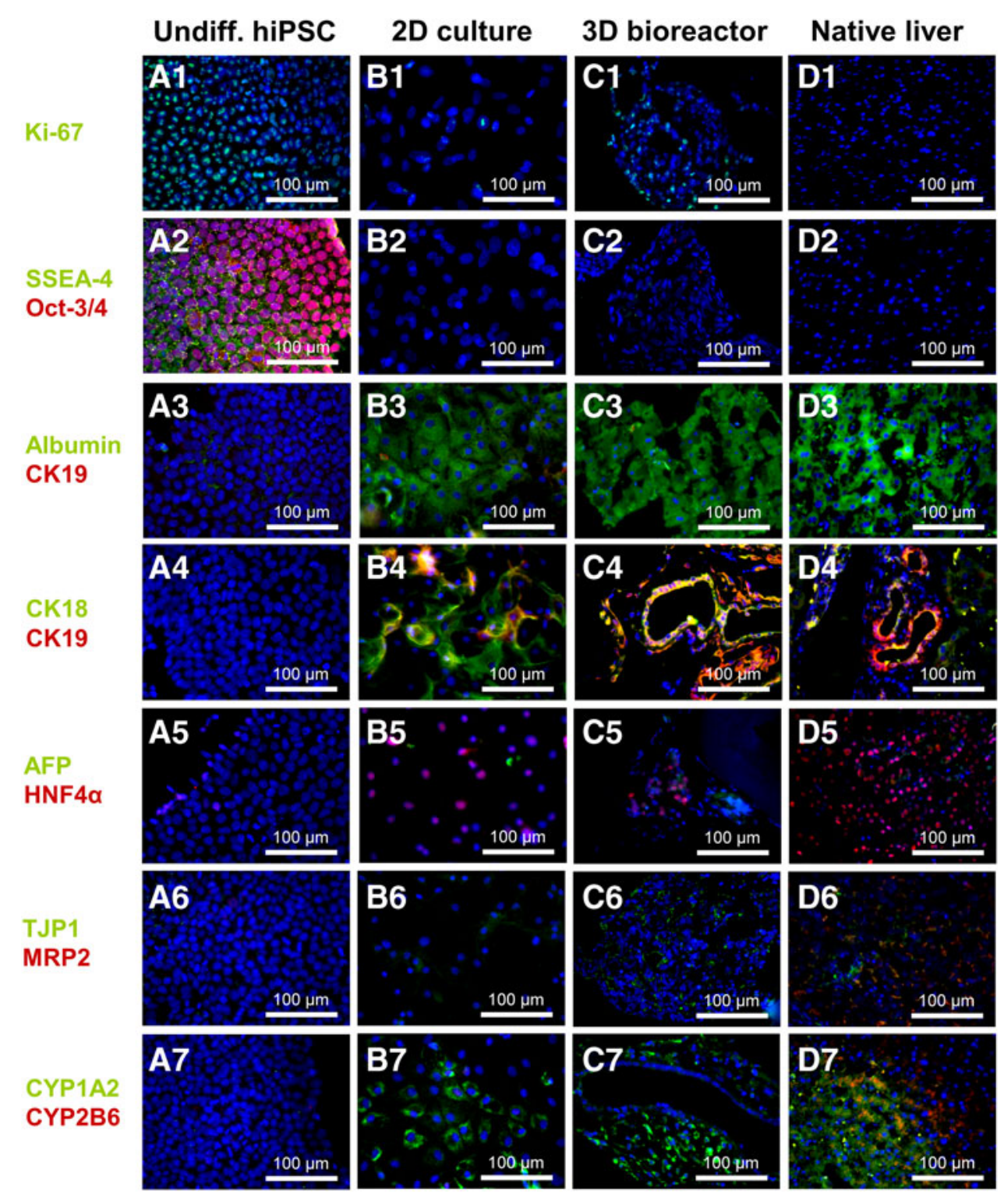

FIG. 4. Immunofluorescence analysis of hiPSC before and after hepatic differentiation in $2 \mathrm{D}$ cultures or 3D bioreactors compared with native human liver tissue. Samples from cultures or liver tissue were stained with (A1D1) Ki-67, (A2-D2) SSEA-4 and Oct-3/4, (A3-D3) albumin and CK19, (A4-D4) CK18 and CK19, (A5-D5) AFP and HNF4 $\alpha$, (A6-D6) TJP1 and MRP2, and with (A7-D7) CYP1A2 and CYP2B6. Nuclei were counterstained with DAPI (blue) or with bisBenzimide H 33342 trihydrochloride in native human liver tissue (blue). CK19, cytokeratin 19. 
approximately two thirds of the cells $(63.6 \% \pm 12.6 \%)$ were proliferating (picture not shown). After hepatic differentiation, around one third of the cells were still proliferating in $2 \mathrm{D}$ cultures $(32.6 \% \pm 20.4 \%$, Fig. $4 \mathrm{~B} 1)$ and $3 \mathrm{D}$ bioreactors $(30.3 \% \pm 18.7 \%$, Fig. $4 \mathrm{C} 1)$. In the native human liver, no Ki-67-positive cells could be detected (Fig. 4D1).

The pluripotency markers SSEA4 and OCT3 were expressed in the majority of undifferentiated hiPSC (Fig. 4A2), but could not be detected in hiPSC-derived HLC in 2D cultures (Fig. 4B2) or 3D bioreactors (Fig. 4C2), or in native human liver tissue (Fig. 4D2). Regarding the expression of hepatocyte-specific markers, the majority of cells was positive for albumin in 2D cultures as well as 3D bioreactors (Fig. 4B3, C3), similar to native human liver tissue (Fig. 4D3).

Cytokeratin 19 (CK19), characteristic for biliary cells, was only detected in a few sparsely distributed cells in 2D cultures (Fig. 4B4), whereas in 3D bioreactors, ring-shaped cell arrangements lined with cells positive for CK18 and CK19 were observed, indicating formation of bile ductlike tubular structures (Fig. 4C4) resembling those occurring in native human liver tissue (Fig. 4D4). Staining for hepatocyte nuclear factor 4-alpha (HNF4A) showed a few positive cells, which were negative for AFP in both 2D cultures and 3D bioreactors (Fig. 4B5, C5). This is in contrast to native human liver tissue where almost all cells showed immunoreactivity for HNF4A (Fig. 4D5).

Staining of tight junction protein (TJP1) and the transporter multidrug resistance-associated protein 2 (MRP2) revealed no immunoreactivity in 2D cultures (Fig. 4B6), while in 3D bioreactors (Fig. 4C6), abundant cells were positive for TJP1 as indicated by thin borders between the cells. In the native liver tissue, most of the cells were double positive for MRP2 and TJP1 (Fig. 4D6).

Immunofluorescence analysis of CYP isoenzymes showed that most of the cells in $2 \mathrm{D}$ cultures and $3 \mathrm{D}$ bioreactors were positive for CYP1A2, but negative for CYP2B6 (Fig. 4B7, C7), which is in line with the functional analysis showing higher activities for CYP1A2. In 3D bioreactors, the inner ring of tubularlike structures lacked CYP1A2 immunoreactivity indicating a zonation of the cells. In native human liver tissue, cells were positive for both CYP isoenzymes (Fig. 4D7). The undifferentiated hiPSC were negative for all liver-specific markers (Fig. 4A3-A7).

\section{Ultrastructural characteristics of hiPSC-derived} differentiated cells

Ultrastructural studies of hiPSC-derived cells after hepatic differentiation in 3D bioreactors showed a hetero- geneous cell population with some similarities to native liver tissue. Cells displaying a high nucleus to cytoplasm ratio indicate the presence of still immature cells (Fig. 5A). In other areas, cells showed microvilli at their apical side with abundant cell-cell contacts consisting of tight junctions and desmosomes indicating cell polarization typical for hepatocytes (Fig. 5B, C). The majority of cells contained numerous mitochondria, which is also a typical feature of hepatocytes due to their high metabolic activity. Distinct interdigitations between neighboring cells and rough endoplasmic reticulum in the cytoplasm were observed in addition to well-developed Golgi apparatuses (Fig. 5D).

\section{Discussion}

Hepatocytes derived from hiPSC represent a promising cell source for pharmacological toxicity testing. Although numerous protocols for the hepatic differentiation of hiPSC exist and are continuously refined, the obtained cells still show a fetal phenotype, ${ }^{16,17}$ and the maturation state of the obtained cells needs to be further improved. In this study, we investigated the potency of a dynamic 3D bioreactor technology to promote the hepatic maturation of hiPSC-derived hepatocytes when compared to conventional $2 \mathrm{D}$ cultures. The differentiation outcome of hiPSC cultured in 2D cultures or 3D bioreactors was analyzed by means of metabolic, phenotypical, and functional parameters.

To allow a direct comparison of the two culture systems, the results for secreted metabolites and proteins as well as for CYP activities were normalized to the initial cell number. Potential proliferation activities of HLC in $2 \mathrm{D}$ cultures or $3 \mathrm{D}$ bioreactors were quantified by means of Ki-67 staining. The protein Ki-67 is expressed during all active phases of the cell cycle (G1, S, G2, mitosis), but is absent in resting cells (G0). ${ }^{35}$ The quantitative analysis of the Ki-67 staining revealed that at the end of hepatic differentiation, there was still mitotic activity. However, since the amount of proliferating cells with $\sim 30 \%$ was comparable in $2 \mathrm{D}$ cultures and $3 \mathrm{D}$ bioreactors, it can be concluded that the observed differences in metabolite/protein secretion and CYP activities are not associated with differences in cell growth.

Glucose consumption rates and lactate production rates as indicators for energy metabolism were doubled in $2 \mathrm{D}$ cultures compared to 3D bioreactors. This could be caused by the formation of $3 \mathrm{D}$ cell aggregates within the bioreactor leading to a gradient and a differential metabolic activity of cells growing either on the surface or in the center of the aggregates. It has been reported that 

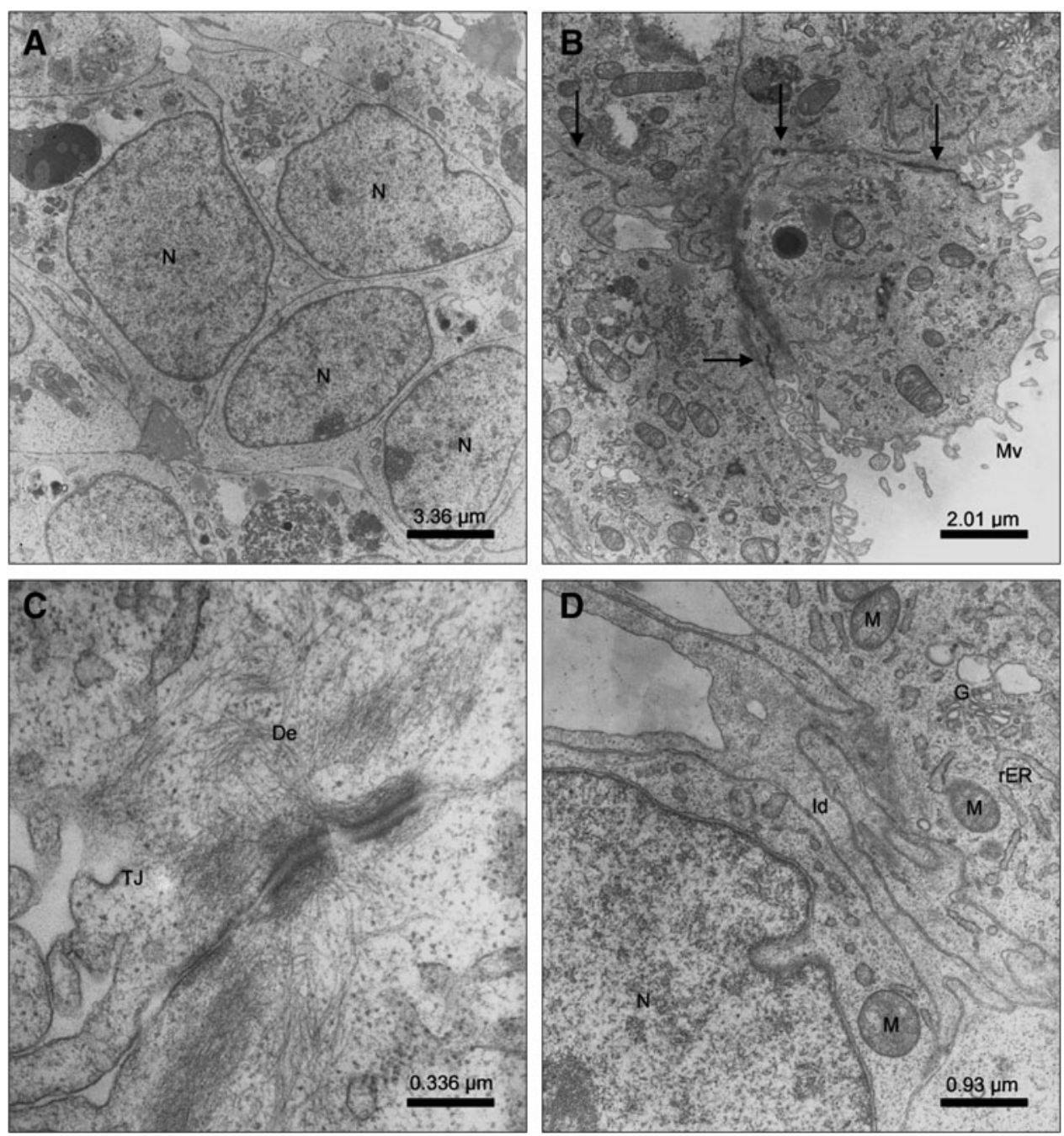

FIG. 5. Ultrastructural characteristics of hiPSC after hepatic differentiation in $3 D$ bioreactors. (A) Cells with a high nucleus:cytoplasm ratio indicating immature cells (N). (B) Cells with distinct microvilli (Mv) and abundant cell-cell contacts (arrows) between neighboring cells. (C) Tight junction (TJ) and desmosome (De) between two neighboring cells. (D) Interdigitations (Id) between two cells with mitochondria (M), rough endoplasmic reticulum (rER), and Golgi apparatus (G).

concentration gradients influence cell differentiation and tissue formation. . $^{36,37}$ Thus, formation of gradients could also be a reason for the heterogeneous cell population observed in $3 \mathrm{D}$ bioreactors as indicated by immunohistochemical analyses and increased GATA2 mRNA expression.

In the described hollow fiber bioreactor, the aggregate size is controlled by the distance between the capillaries. In other bioreactor technologies such as stirred tank vessels, the aggregate size is influenced by the impeller design ${ }^{38}$ and the stirring velocity, ${ }^{39}$ but stirring can cause shear stress and cell damage. ${ }^{40}$ Another bioreactor technology with minimized shear stress is the rotating-wall vessel, which consists of a horizontally rotating cylindrical culture vessel with a coaxial tubular oxygenator avoiding nutrient compartmentalization and barrier formation. ${ }^{41}$

Furthermore, a significantly lower LDH release was detected in 3D bioreactors compared with 2D cultures. One possible reason could be a protective effect of $3 \mathrm{D}$ cell aggregates against damage caused by the applied activin $\mathrm{A}$, which can induce apoptosis. ${ }^{42}$ However, $\mathrm{LDH}$ is released during both necrosis and apoptosis ${ }^{43}$ and therefore does not allow distinguishing between necrosis and apoptosis. An apoptosis assay would be 
needed to clarify whether the observed LDH release is due to apoptosis processes.

AFP expression and secretion were detected in HLC in both 2D cultures and 3D bioreactors, with significantly higher secretion rates in 2D cultures. In contrast, albumin secretion showed a distinctly higher increase in $3 \mathrm{D}$ cultures than in $2 \mathrm{D}$ cultures. In vivo AFP is only expressed during liver embryogenesis, in fetal liver cells, and hepatocellular carcinomas, ${ }^{44}$ while albumin is produced by differentiated hepatocytes in the adult liver. Thus, the findings of $A F P$ and $A L B$ expression indicate a higher maturation grade of HLC in 3D bioreactors than in 2D cultures.

Expression of AFP in HLC in association with expression of markers for mature hepatocytes such as $A L B$ and various $C Y P$ isoenzymes has been described in several studies. ${ }^{7,45,46}$ Gieseck et al. ${ }^{47}$ also observed lower AFP secretion rates and higher albumin secretion rates in $3 \mathrm{D}$ clump cultures compared to $2 \mathrm{D}$ cultures, which emphasizes the value of $3 \mathrm{D}$ culture systems to promote hepatic maturation of hiPSC. Since albumin secretion was still increasing at the end of hepatic differentiation, a further maturation could be achieved by prolongation of the differentiation period.

This finding is supported by the observed increase of urea secretion in $3 \mathrm{D}$ bioreactors. Urea is the major end product of protein nitrogen metabolism and is synthesized by the urea cycle in the liver from ammonia. Hence, urea secretion is an important functional marker for mature hepatocytes. To further validate results from urea secretion, the expression of enzymes from the urea cycle such as carbamoyl phosphate synthetase I or transcarbamylase could be analyzed.

In addition to AFP and SOX17 as markers for both, definitive and extraembryonic endoderm, SOX7 was analyzed as a specific marker for extraembryonic endoderm. The gene expression of SOX7 was similar in HLC in $2 \mathrm{D}$ cultures, in $3 \mathrm{D}$ bioreactors, and in $\mathrm{PHH}$, indicating that the hiPSC-derived HLC did not contain significant amounts of extraembryonic cells, which confirms the hepatic origin of AFP and also of SOX17 in this study. According to the Human Protein Atlas, the SOX7 expression is strongest in the placenta, but a weak expression is also found in the human liver. ${ }^{48}$

The ability to metabolize drugs through specific CYP isoenzymes is crucial for potential application of hiPSCderived HLC in pharmacological studies. Functional activities of CYP1A2 and CYP3A4 were detected in both 2D cultures and 3D bioreactors, while the CYP2B6 activity was observed only in 3D bioreactors. However, all CYP isoenzyme activities were significantly lower in HLC than in
PHH. Data from other studies also showed a lower expression and activity of CYPs in differentiated hiPSC compared to $\mathrm{PHH} .{ }^{5,49}$ However, since different experimental conditions were used in those studies, a direct comparison of CYP activities from different publications is difficult.

Measurements of mRNA expression levels revealed that the pluripotency markers POU5F1 and NANOG were stronger downregulated in $2 \mathrm{D}$ cultures than in $3 \mathrm{D}$ bioreactors. This could be explained by the formation of an activin A gradient within the 3D cell aggregates. Activin A recapitulates the nodal signaling pathway in vitro, which stimulates DE derivation from pluripotent stem cells. ${ }^{50}$ In lower concentrations, activin $\mathrm{A}$ is used to maintain pluripotency of hiPSC ${ }^{51}$ and hESC. ${ }^{52}$ This could imply that DE formation is decreased in the center of the cell aggregates and other factors such as dimethylsulfoxide (DMSO) might be required to block the effect of activin $\mathrm{A}$ on the pluripotency. ${ }^{53}$

On the other hand, the formation of gradients of activin $\mathrm{A}$ and other factors might also promote $3 \mathrm{D}$ tissue formation from HLC by reflecting the physiological situation in the liver lobule, which is characterized by an oxygen gradient between the periportal and the perivenous area. This is supported by immunohistochemical and TEM pictures showing tissue-like organization of the cells within $3 \mathrm{D}$ bioreactors, including bile duct-like structures characterized by CK18/CK19 double staining. Miki et al. ${ }^{26}$ also observed the formation of bile duct-like structures after hepatic differentiation of hESC in the 3D bioreactor. Bile ducts are lined with cholangiocytes, which, like the hepatocytes, originate from the bipotent hepatoblasts. ${ }^{54}$

Current protocols for direct differentiation of pluripotent stem cells to cholangiocytes and their in vitro generation from hepatoblasts are to some extent similar to protocols used for hepatocyte differentiation since they use factors such as epidermal growth factor, insulin, and hydrocortisone. ${ }^{55,56}$ De Assuncao et al. ${ }^{55}$ observed that cholangiocyte markers already became apparent during the hepatic progenitor phase, which would explain the appearance of cells positive for CK19 in this study.

In addition, abundant tight junctions were detected by immunohistochemical staining and ultrastructural analysis using TEM. The presence of tight junctions is a prerequisite for the formation of bile canaliculi as a characteristic feature of differentiated hepatocytes. In contrast to tight junction protein, the transporter protein MRP2, which is expressed in the canalicular (apical) membrane area of the hepatocyte and contributes to biliary transport, was negative both in $2 \mathrm{D}$ cultures and $3 \mathrm{D}$ bioreactor cultures, indicating incomplete cell polarization. 
The detailed comparison of hiPSC-derived HLC with $\mathrm{PHH}$ showed that further improvement of HLC maturation is needed. This could be achieved by combining other promising differentiation strategies with the $3 \mathrm{D}$ bioreactor culture: for example, differentiation into the mesodermal direction during DE differentiation, detected by GATA2 expression in the 3D bioreactor, could be excluded by adding specific inhibitors ${ }^{57}$ and cocultivation with primitive endothelial cells or mesenchymal stem cells could recapitulate the in vivo organogenesis even more closely in the $3 \mathrm{D}$ culture. ${ }^{15}$ In addition, Kim et al. ${ }^{46}$ could show that repeated stimulation of HLC with xenobiotics could improve their metabolizing activity.

A further strategy is based on the usage of an hiPSC line, which is derived from primary hepatoblasts as already shown for mouse iPSC. ${ }^{58}$ This could also improve the hepatic differentiation since recent studies suggest that iPSC retain a residual donor cell memory, which may impact their capacity to differentiate into the cell type of origin. ${ }^{59}$

\section{Conclusion}

In conclusion, the hepatic maturation of hiPSC-derived HLC was improved in 3D bioreactors compared with $2 \mathrm{D}$ cultures in terms of albumin secretion, CYP2B6 activity, and formation of tissue-like structures with cell-cell contacts. However, some aspects of hepatic functionality were still insufficient, for example, $A F P$ was still expressed, and the activity of CYP2C9 and expression of the biliary transporter MRP2 could not be detected. The 3D bioreactor could be used to investigate approaches to improve the maturation of hiPSCderived hepatocytes and generate fully differentiated hepatocytes from hiPSC in a physiological-like 3D environment in the future.

\section{Acknowledgments}

The research leading to these results has received support from the Innovative Medicines Initiative Joint Undertaking under grant agreement no. 115439, resources of which are composed of financial contribution from the European Union's Seventh Framework Programme (FP7/2007-2013) and EFPIA companies' in kind contribution. This article reflects only the author's views and neither the IMI JU, EFPIA, nor the European Commission is liable for any use that may be made of the information contained therein.

\section{Author Disclosure Statement}

No competing financial interests exist.

\section{References}

1. Kola I, Landis J. Can the pharmaceutical industry reduce attrition rates? Nat Rev Drug Discov. 2004;3:711-715.

2. Lewis DF, loannides C, Parke DV. Cytochromes P450 and species differences in xenobiotic metabolism and activation of carcinogen. Environ Health Perspect. 1998;106:633-641.

3. Giri S, Bader A. Improved preclinical safety assessment using micro-BAL devices: the potential impact on human discovery and drug attrition. Drug Discov Today. 2011;16:382-397.

4. Ingelman-Sundberg M. Pharmacogenetics of cytochrome P450 and its applications in drug therapy: the past, present and future. Trends Pharmacol Sci. 2004;25:193-200.

5. Takayama K, Morisaki Y, Kuno S, et al. Prediction of interindividual differences in hepatic functions and drug sensitivity by using human iPSderived hepatocytes. Proc Natl Acad Sci U S A. 2014;111:16772-16777.

6. Hay DC, Zhao D, Ross A, et al. Direct differentiation of human embryonic stem cells to hepatocyte-like cells exhibiting functional activities. Cloning Stem Cells. 2007;9:51-62.

7. Sullivan GJ, Hay DC, Park IH, et al. Generation of functional human hepatic endoderm from human induced pluripotent stem cells. Hepatology. 2010:51:329-335.

8. Hannan NR, Segeritz CP, Touboul T, et al. Production of hepatocyte-like cells from human pluripotent stem cells. Nat Protoc. 2013;8:430-437.

9. Szkolnicka D, Farnworth SL, Lucendo-Villarin B, et al. Deriving functional hepatocytes from pluripotent stem cells. Curr Protoc Stem Cell Biol. 2014;30:1G.5.1-12.

10. Medine CN, Lucendo-Villarin B, Storck C, et al. Developing high-fidelity hepatotoxicity models from pluripotent stem cells. Stem Cells Transl Med. 2013;2:505-509.

11. Sjogren AK, Liljevald M, Glinghammar B, et al. Critical differences in toxicity mechanisms in induced pluripotent stem cell-derived hepatocytes, hepatic cell lines and primary hepatocytes. Arch Toxicol. 2014;88:1427-1437.

12. Schwartz RE, Trehan $K$, Andrus $L$, et al. Modeling hepatitis $C$ virus infection using human induced pluripotent stem cells. Proc Natl Acad Sci U S A. 2012;109:2544-2548.

13. Shlomai A, Schwartz RE, Ramanan V, et al. Modeling host interactions with hepatitis $B$ virus using primary and induced pluripotent stem cell-derived hepatocellular systems. Proc Natl Acad Sci U S A. 2014;111:12193-12198.

14. $\mathrm{Ng} \mathrm{S}$, Schwartz RE, March S, et al. Human iPSC-derived hepatocyte-like cells support Plasmodiumliver-stage infection in vitro. Stem Cell Reports. 2015;4:348-359.

15. Takebe T, Sekine $K$, Enomura M, et al. Vascularized and functional human liver from an iPSC-derived organ bud transplant. Nature. 2013;499:481-484.

16. Baxter $M$, Withey $S$, Harrison $S$, et al. Phenotypic and functional analyses show stem cell-derived hepatocyte-like cells better mimic fetal rather than adult hepatocytes. J Hepatol. 2015;62:581-589.

17. Godoy P, Schmidt-Heck W, Natarajan K, et al. Gene networks and transcription factor motifs defining the differentiation of stem cells into hepatocyte-like cells. J Hepatol. 2015;63:934-942.

18. Kern A, Bader A, Pichlmayr R, et al. Drug metabolism in hepatocyte sandwich cultures of rats and humans. Biochem pharmacol. 1997;54:761-772.

19. Tuschl G, Hrach J, Walter Y, et al. Serum-free collagen sandwich cultures of adult rat hepatocytes maintain liver-like properties long term: a valuable model for in vitro toxicity and drug-drug interaction studies. Chem Biol Interact. 2009;181:124-137.

20. Schyschka L, Sánchez JJM, Wang Z, et al. Hepatic 3D cultures but not 2D cultures preserve specific transporter activity for acetaminophen-induced hepatotoxicity. Arch Toxicol. 2013;87:1581-1593.

21. Powers MJ, Janigian DM, Wack KE, et al. Functional behavior of primary rat liver cells in a three-dimensional perfused microarray bioreactor. Tissue Eng. 2002;8:499-513.

22. Mazzei D, Guazzardi S. A low shear stress modular bioreactor for connected cell culture under high flow rates. Bioctechnol Bioeng. 2010;106:127-137.

23. Tilles AW, Baskaran $H$, Roy $P$, et al. Effects of oxygenation and flow on the viability and function of rat hepatocytes cocultured in a microchannel flat-plate bioreactor. Biotechnol Bioeng. 2015;73:379-389.

24. Hoffmann SA, Müller-Vieira U, Biemel K, et al. Analysis of drug metabolism activities in a miniaturized liver cell bioreactor for use in pharmacological studies. Biotechnol Bioeng. 2012;109:3172-3181.

25. Lübberstedt M, Müller-Vieira U, Biemel KM, et al. Serum-free culture of primary human hepatocytes in a miniaturized hollow-fibre membrane 
bioreactor for pharmacological in vitro studies. J Tissue Eng Regen Med. 2015;9:1017-1026.

26. Miki T, Ring A, Gerlach J. Hepatic differentiation of human embryonic stem cells is promoted by three-dimensional dynamic perfusion culture conditions. Tissue Eng Part C Methods. 2011;17:557-568.

27. Stachelscheid $\mathrm{H}$, Wulf-Goldenberg A, Eckert $\mathrm{K}$, et al. Teratoma formation of human embryonic stem cells in three-dimensional perfusion culture bioreactors. J Tissue Eng Regen Med. 2013;7:729-741.

28. Zeilinger K, Schreiter T, Darnell M, et al. Scaling down of a clinical 3D perfusion multi-compartment hollow fiber liver bioreactor developed for extracorporeal liver support to an analytical scale device useful for hepatic pharmacological in vitro studies. Tissue Eng Part C Methods. 2011;17:549-556.

29. $\mathrm{Yu}$ J, Hu K, Smuga-Otto K, et al. Human induced pluripotent stem cells free of vector and transgene sequences. Science. 2009;324:797-801.

30. Hay DC, Zhao D, Fletcher J, et al. Efficient differentiation of hepatocytes from human embryonic stem cells exhibiting markers recapitulating liver development in vivo. Stem Cells. 2008;26:894-902.

31. Hay DC, Fletcher J, Payne C, et al. Highly efficient differentiation of hESCs to functional hepatic endoderm requires Activin A and Wnt3a signaling. Proc Natl Acad Sci U S A. 2008;105:12301-12306.

32. Pfeiffer $\mathrm{E}$, Kegel $\mathrm{V}$, Zeilinger $\mathrm{K}$, et al. Featured article: isolation, characterization, and cultivation of human hepatocytes and non-parenchymal liver cells. Exp Biol Med (Maywood). 2015;240:645-656.

33. Knöspel F, Freyer N, Stecklum M, et al. Periodic harvesting of embryonic stem cells from a hollow-fiber membrane based four-compartment bioreactor. Biotechnol Prog. 2016;32:141-151.

34. Livak KJ, Schmittgen TD. Analysis of relative gene expression data using real-time quantitative PCR and the 2(-Delta Delta C(T)) Method. Methods. 2001;25:402-408.

35. Scholzen T, Gerdes J. The Ki-67 protein: from the known and the unknown. J Cell Physiol. 2000;182:311-322.

36. Singh M, Berkland C, Detamore MS. Strategies and applications for incorporating physical and chemical signal gradients in tissue engineering. Tissue Eng Part B Rev. 2008;14:341-366.

37. Uzel SG, Amadi OC, Pearl TM, et al. Simultaneous or sequential orthogonal gradient formation in a $3 \mathrm{D}$ cell culture microfluidic platform. Small. 2016;12:612-622.

38. Olmer R, Lange A, Selzer S, et al. Suspension culture of human pluripotent stem cells in controlled, stirred bioreactors. Tissue Eng Part C Methods. 2012;18:772-784.

39. Storm MP, Orchard CB, Bone HK, et al. Three-dimensional culture systems for the expansion of pluripotent embryonic stem cells. Biotechnol Bioeng. 2010;107:683-695

40. Kinney MA, Sargent CY, McDevitt TC. The multiparametric effects of hydrodynamic environments on stem cell culture. Tissue Eng Part B Rev. 2011:17:249-262.

41. Hammond TG, Hammond JM. Optimized suspension culture: the rotating-wall vessel. Am J Physiol Renal Physiol 2001;281:F12-F25.

42. Chen YG, Wang $Q$, Lin SL, et al. Activin signaling and its role in regulation of cell proliferation, apoptosis, and carcinogenesis. Exp Biol Med (Maywood). 2006;231:534-544.

43. Denecker G, Vercammen D, Steemans M, et al. Death receptor-induced apoptotic and necrotic cell death: differential role of caspases and mitochondria. Cell Death Differ. 2001;8:829-840.

44. Terentiev AA, Moldogazieva NT. Alpha-fetoprotein: a renaissance. Tumour Biol. 2013;34:2075-2091.

45. Cameron K, Tan R, Schmidt-Heck W, et al. Recombinant laminins drive the differentiation and self-organization of hESC-derived hepatocytes. Stem Cell Reports. 2015;5:1250-1262.

46. Kim JH, Jang YJ, An SY, et al. Enhanced metabolizing activity of human ES cell-derived hepatocytes using a 3D culture system with repeated exposures to xenobiotics. Toxicol Sci. 2015;147:190-206.

47. Gieseck RL 3rd, Hannan NR, Bort R, et al. Maturation of induced pluripotent stem cell derived hepatocytes by 3D-culture. PLoS One. 2014;9:e86372.

48. Uhlén $M$, Fagerberg $L$, Hallström BM, et al. Tissue-based map of the human proteome. Science. 2015;347:1260419.

49. Ek M, Söderdahl T, Küppers-Munther B, et al. Expression of drug metabolizing enzymes in hepatocyte-like cells derived from human embryonic stem cells. Biochem Pharmacol. 2007;74;496-503.
50. D'Amour KA, Agulnick AD, Eliazer S, et al. Efficient differentiation of human embryonic stem cells to definitive endoderm. Nat Biotechnol. 2005;23:1534-1541.

51. Tomizawa $M$, Shinozaki $F$, Sugiyama $T$, et al. Activin $A$ is essential for feeder-free culture of human induced pluripotent stem cells. J Cell Biochem. 2013:114:584-588.

52. Beattie GM, Lopez AD, Bucay N, et al. Activin A maintains pluripotency of human embryonic stem cells in the absence of feeder layers. Stem Cells. 2005;23:489-495

53. Czysz K, Minger S, Thomas N. DMSO efficiently down regulates pluripotency genes in human embryonic stem cells during definitive endoderm derivation and increases the proficiency of hepatic differentiation. PLoS One. 2015;10:e0117689.

54. Zhang Y, Bai XF, Huang CX. Hepatic stem cells: existence and origin. World J Gastroenterol. 2003;9:201-204.

55. De Assuncao TM, Sun Y, Jalan-Sakrikar N, et al. Development and characterization of human-induced pluripotent stem cell-derived cholangiocytes. Lab Invest. 2015;95:684-696.

56. Dianat N, Dubois-Pot-Schneider H, Steichen C, et al. Generation of func tional cholangiocyte-like cells from human pluripotent stem cells and HepaRG cells. Hepatology. 2014;60:700-714.

57. Loh KM, Ang LT, Zhang J, et al. Efficient endoderm induction from human pluripotent stem cells by logically directing signals controlling lineage bifurcations. Cell Stem Cell. 2014;14:237-252.

58. Lee SB, Seo D, Choi D, et al. Contribution of hepatic lineage stage-specific donor memory to the differential potential of induced mouse pluripotent stem cells. Stem Cells. 2012;30:997-1007.

59. Vaskova EA, Stekleneva AE, Medvedev SP, et al. "Epigenetic memory" phenomenon in induced pluripotent stem cells. Acta Naturae. 2013;5:15-21.

Cite this article as: Freyer N, Knöspel F, Strahl N, Amini L, Schrade $P$, Bachmann S, Damm G, Seehofer D, Jacobs F, Monshouwer M, Zeilinger K (2016) Hepatic differentiation of human induced pluripotent stem cells in a perfused three-dimensional multicompartment bioreactor, BioResearch Open Access 5:1, 235-248, DOI: 10.1089/biores .2016.0027.

\section{Publish in BioResearch Open Access}

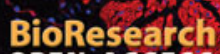
OPEN ACCESS

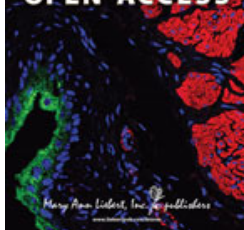
- Immediate, unrestricted online access - Rigorous peer review - Compliance with open access mandates - Authors retain copyright - Highly indexed - Targeted email marketing 